\title{
GA-Based Selection of Vaginal Microbiome Features Associated with Bacterial Vaginosis
}

\author{
Joi Carter \\ North Carolina A\&T State University \\ Department of Computer Science \\ Greensboro, NC USA \\ jncarte1@ncat.edu
}

\author{
Daniel Beck \\ University of Idaho \\ Bioinformatics \& Comp. Biology \\ Moscow, ID USA \\ beck5429@vandals.uidaho.edu
}

\author{
Henry Williams \\ North Carolina A\&T State University \\ Department of Computer Science \\ Greensboro, NC USA \\ hwilliams18@gmail.com
}

\author{
Gerry Dozier \\ North Carolina A\&T State University \\ Department of Computer Science \\ Greensboro, NC USA \\ gvdozier@ncat.edu
}

\author{
James A. Foster \\ University of Idaho \\ Biological Sciences \\ Moscow, ID USA \\ foster@uidaho.edu
}

\begin{abstract}
In this paper, we successfully apply GEFeS (Genetic \& Evolutionary Feature Selection) to identify the key features in the human vaginal microbiome and in patient meta-data that are associated with bacterial vaginosis (BV). The vaginal microbiome is the community of bacteria found in a patient, and meta-data include behavioral practices and demographic information. Bacterial vaginosis is a disease that afflicts nearly one third of all women, but the current diagnostics are crude at best. We describe two types of classifies for BV diagnosis, and show that each is associated with one of two treatments. Our results show that the classifiers associated with the 'Treat Any Symptom' version have better performances that the classifier associated with the 'Treat Based on N-Score Value'. Our long term objective is to develop a more accurate and objective diagnosis and treatment of BV.
\end{abstract}

\section{Categories and Subject Descriptors}

J.3 [Life and Medical Sciences]: Health

\section{General Terms}

Algorithms

\section{Keywords}

Bacterial Vaginosis, GEFeS, Genetic Algorithm, Human Microbiome

\section{INTRODUCTION}

Human beings have coevolved with bacteria. $90 \%$ of the cells in our bodies, and $99 \%$ of the genes, are bacterial rather than human. This "human microbiome" affects human health. Examples include the adult gut microbiome and obesity[1,2], the neonatal gut microbiome and sepsis[17], the lung microbiome and pulmonary infections[3] and cystic fibrosis[18], and the vaginal

Permission to make digital or hard copies of all or part of this work for personal or classroom use is granted without fee provided that copies are not made or distributed for profit or commercial advantage and that copies bear this notice and the full citation on the first page. To copy otherwise, or republish, to post on servers or to redistribute to lists, requires prior specific permission and/or a fee.

GECCO'14, July 12-16, 2014, Vancouver, BC, Canada.

Copyright (C) 2014 ACM 978-1-4503-2662-9/14/07...\$15.00. microbiome and bacterial vaginosis[4-6]. All known microbiomeassociated diseases appear to be forms of dysbiosis, an ecological disturbance of the "normal" bacterial community in an individual, rather than the emergence of a pathogen. Therefore, the challenge to diagnosing such diseases is to uncover the features of a complex mélange of bacteria whose variation is associated with those diseases. This paper presents a new genetic algorithm, GEFeS (Genetic \& Evolutionary Feature Selection), originally developed for image feature recognition, that successfully reveals the changes in the vaginal microbiome associated with bacterial vaginosis (BV), extending previous work on machine learning classifiers for BV [14].

BV affects nearly one third of all women at any given time[11], and is associated with increased risk for some sexually transmitted diseases and preterm birth. There are two clinical diagnostics for BV. The Amsel criteria (Any-Symptom approach) searches for the presence of at least three of the following criteria: the presence of discharge, a positive whiff test, the presence of clue cells, and a $\mathrm{pH}$ greater than 4.5[12]. The Nugent score $(\mathrm{N}-$ Score approach), a scale of 0-10 with anything over 7 indicating disease, is based counting gram-positive cells with morphologies similar to some Lactobacillus species (large rods) [13].

Both diagnostic criteria are problematic, resulting in up to $30 \%$ of BV diagnoses being for women who in fact have no observable symptoms [19]. Misdiagnosis can have serious adverse health consequences, since the prescribed treatment is systemic antibiotics, which would seem unnecessary, if not unethical, when there are no symptoms to treat. Both diagnostic tests assume that the predominance of Lactobacillus species indicates vaginal health, and their absence indicates disease, on the assumption that $\mathrm{pH}$ maintenance is the primary mechanism for maintaining health. However, different Lactobacillus species have very different mechanisms for acidification, and there are other species that provide the same function. More significantly, recent cultivation independent surveys of the vaginal microbiome have shown that healthy women, especially in minority communities, do not have Lactobacillus dominated vaginal communities $[8,10]$ and that the "normal" vaginal micobiome varies significantly from day to day[15,20,21]. The two criteria frequently disagree, usually with the Nugent score indicating BV when Amsel does not. Amsel is 
the most commonly used diagnostic, since it is more amenable to a clinical setting. However, the "sniff test" is subjective, there are no "clue cells" that unambiguously identify BV, and the average $\mathrm{pH}$ of healthy black and Hispanic women are $4.7 \pm 1.04$ and $5.0 \pm 0.74$ respectively [15]. It would be helpful to have a diagnostic tool based on a small set of objective features, recognizing the dependence of $\mathrm{BV}$ on the complicated vaginal microbiome and characteristics of individual patients.

Our feature extraction algorithm shows that such an objective, symptomatic and microbiome-based diagnostic is possible. It takes as input a list of the relative abundances of all observed bacterial populations in the vaginal microbiome along with patient metadata such as demographics and sexual practices. Relative abundances are most accurately determined by a cultivation independent assay of a hypervariable regions of the $16 \mathrm{~S}$ rRNA gene, obtained via next generation sequencing. The technique is to extract all DNA from a sample, amplify and sequence the target rRNA sequences, and then cluster the sequences into " operational taxonomical units" (OTUs). Standard practice treats OTUs clustered at 3\% similarity as separate species, naming them by searching a database of all known sequences..

In this paper, we use GEFeS to evolve high-performance feature subsets in order to develop classifiers for determining whether a subject has BV based on: the Any-Symptom (Amsel criteria) approach or the N-Score (Nugent score) approach. The remainder of this paper is as follows. In Section II, we provide a brief overview of GEFeS. In Section III, describe our experiment and in Section IV we present our results. In Section V, we present our conclusions and future work

\section{GEFES}

GEFeS [25] has been successfully used for feature selection on a variety of recognition and classification problems. GEFeS evolves a population of candidate feature masks (FMs). A candidate FM, fmi, can be viewed as a triple, $<\mathrm{Mi}$, ti, fi $>$, where Mi is set of $\mathrm{N}$ masks, $\mathrm{Mi}=\{\mathrm{mi}, 0, \mathrm{mi}, 1, \ldots$, mi,n- 1$\}$ where each $\mathrm{mi}, \mathrm{j} \quad[0 . .1]$ is associated with feature $\mathrm{j}$ in the original (baseline) feature set, where ti [0.1] is a masking threshold value, and fi is the fitness of the candidate FM, fmi . If mi,j $<$ ti, then the $j$ th feature of the original feature set is masked. GEFeS co-evolves the Mi and ti of candidate FMs, fmi .

GEFeS incorporates cross-validation in order to evolve FMs that generalize well. The evolutionary process is as follows. Initially, a random population of candidate FMs. Each candidate FM is then evaluated using the training set of feature templates and assigned a fitness that corresponds to the number of errors the candidate FM causes. Additionally, each candidate FM is evaluated using a validation set of feature templates. The best performing FM on the validation set is saved as FM*. After the initially population has been created and evaluated, two candidate FMs are selected and mated to form an offspring FM. The offspring FM is then evaluated and assigned a fitness value as above. The offspring FM is then evaluated using the validation set. If the offspring FM causes fewer errors than the current $\mathrm{FM}^{*}$, then it becomes the new FM*. This process is repeat for a userspecified number of candidate FM evaluations, after which $\mathrm{FM}^{*}$ is returned to the user. Figure 1 provides an overview of the evolutionary process of GEFeS.

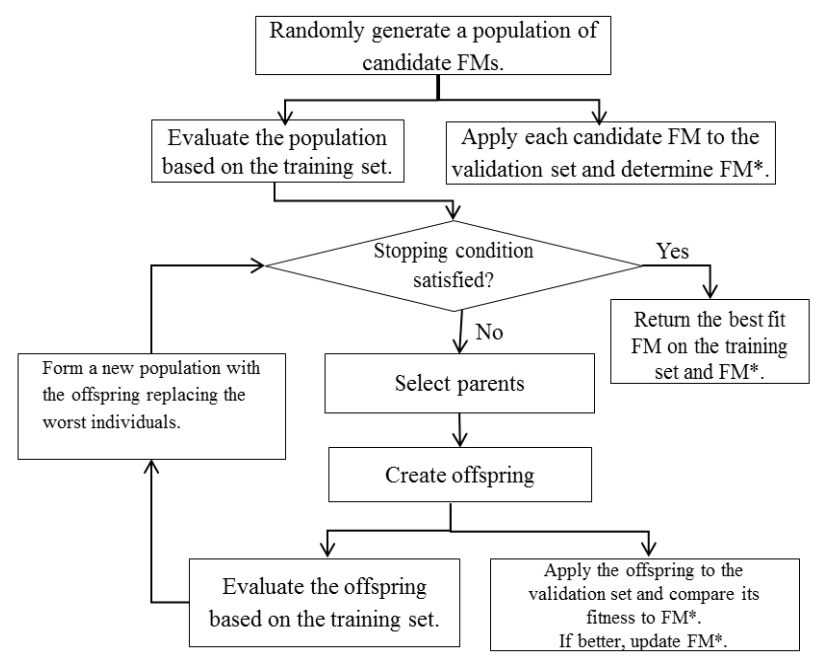

Figure 1. The Evolutionary Process of GEFe S

\section{EXPERIMENT}

The dataset used for our experiment consisted of 1601 instances with 410 features. The data include relative abundances of bacterial species determined by next-generation sequencing of $16 \mathrm{~S}$ rRNA fingerprint sequences, demographic information, and behavioral and health data from daily journals from 25 women over a 10 week period[22]. The study received Institutional Review Board approval from the University of Maryland School of Medicine and sample collection followed the protocols from earlier studies[8,23,24]. This dataset was divided into a training set consisting of $60 \%$ of instances, a validation set consisting of $20 \%$ of the instances, and a test set that consisted of $20 \%$ of the instances. The objective of this experiment was to evolve FMs that generalized well to unseen instances.

In comparing two instances, the Manhattan distance between a probe and gallery feature (sub)vectors used calculated. After a probe instance has been compared to each gallery instance, the gallery instance that is closest to the probe is considered its mate. If the gallery instance came from a different sample than the probe instance, an error is recorded.

\section{RESULTS}

The results presented in this paper were created by applying GEFeS in an effort to evolve high-performance subsets of features associated with the N-Score and Any-Symptom BV problems. GEFeS is an instance of a steady-state GA found in the XTOOLSS suite of Evolutionary Computations [25]. GEFeS evolved a population of 20 candidate FMs, used uniform crossover, a mutation usage rate of 1.0, and Gaussian mutation of the form $0.2 \mathrm{~N}(0,1)$. GEFeS was run a total of 30 times for each of three stopping conditions, 2000, 4000, and 8000 function (candidate FM) evaluations, for a total of 90 runs on both the NScore and Any-Symptom BV Problems.

In Table 1, the performances of GEFeS at 2000, 4000, and 8000 function evaluations are compared with the full feature sets (baseline) for the N-Score and Any-Symptom problems. The first column in Table 1 denotes the two methods used GEFeSnScore and GEFeSanySymptom, the second column the corresponding 
baseline accuracies of the full features set, and the 3rd, 4th, and 5 th column are the average accuracies of the feature subsets.

Table 1: Comparison of the Performances of the Baseline Features Sets vs. the GEFeS Feature Sets for the N-Score and Any-Symptom BV Problems wrt Average Accuracy

\begin{tabular}{|l|l|l|l|l|}
\hline Method & B & 2000FE & 4000FE & 8000FE \\
\hline GEFeS $_{\text {nScore }}$ & 0.9252 & 0.9756 & 0.9764 & 0.9773 \\
\hline GEFeS $_{\text {anySymptom }}$ & 0.9626 & 0.9945 & 0.9954 & 0.9953 \\
\hline
\end{tabular}

The average performances of the evolved feature subsets at 2000, 4000, and 8000 function evaluations outperforms the performances of the baseline feature sets. On average, GEFeSanySymptom is a superior criterion to GEFeSnScore. The performance using GEFeSnScore increases with the number of evaluations in all cases, and using GEFeSanySymptom performance increases initially

Table 2 compares the average percentage of he full feature sest required for classification for both diagnostic criteria. On average, both criteria use reduced feature sets. The required number of features varies with the number of iterations.

Table 2: Comparison of the Performances of the Baseline Features Sets vs. the GEFeS Feature Sets for the N-Score and Any-Symptom BV Problems wrt the Average Percentage of Features Needed.

\begin{tabular}{|l|l|l|l|l|}
\hline Method & B & 2000FE & 4000FE & 8000FE \\
\hline GEFeS $_{\text {nScore }}$ & $100 \%$ & $44.56 \%$ & $42.82 \%$ & $32.45 \%$ \\
\hline GEFeS $_{\text {anySymptom }}$ & $100 \%$ & $46.87 \%$ & $47.93 \%$ & $47.00 \%$ \\
\hline
\end{tabular}

\section{CONCLUSIONS \& FUTURE WORK}

In this paper we show that GA-based feature selection can increase classification accuracy of $\mathrm{BV}$ from microbiome data using fewer features, for either diagnostic criterion. But AnySymptom induces a better baseline performance. Future research will expand this project using X-TOOLSS to compare GEFeS to other evolutionary techniques.

Future work includes a detailed analysis of the actual features that GEFeS discovered to be associated with BV. Since these features include both microbial and patient behaviors, they should be evaluated from both microbial ecology and clinical perspectives; proposing hypotheses about how specific collection of bacterial species or patient demographics and behavior contribute to health or disease, and how applicable these findings are to diagnostic evaluation in clinical settings (which rarely have access to next generation sequencing).

\section{ACKNOWLEDGMENTS}

Funded by NSF DBI0939454 and NIH P20GM016454. We thank Roxanne Hickey, Larry J. Forney, and Jacques Ravel for substantial help and for the data for this study.

\section{REFERENCES}

[1] Ley RE, Turnbaugh PJ, Klein S, Gordon JI. (2006) Microbial ecology: Human gut microbes associated with obesity. Nature 444: 1022-1023

[2] Turnbaugh PJ, Ley RE, Mahowald MA, Magrini V, Mardis ER, Gordon JI. (2006) An obesity-associated gut microbiome with increased capacity for energy harvest. Nature 444: 1027-1031

[3] Sibley CD, Parkins MD, Rabin HR, Duan K, Norgaard JC, Surette MG. (2008) A polymicrobial perspective of pulmonary infections exposes an enigmatic pathogen in cystic fibrosis patients. Proceedings of the National Academy of Science USA, 105: 15070-15075.

[4] Ling Z, Kong J, Liu F, Zhu H, Chen X, et al. (2010) Molecular analysis of the diversity of vaginal microbiota associated with bacterial vaginosis. BMC Genomics 11: 488

[5] Srinivasan S, Fredricks DN. (2008) The human vaginal bacterial biota and bacterial vaginosis. Interdisciplinary Perspectives on Infectious Diseases 2008

[6] Brotman RM. (2011) Vaginal microbiome and sexually transmitted infections: an epidemiologic perspective. Journal of Clinical Investigation 121: 4610-4617

[7] Amann RI, Ludwig W, Schleifer KH (1995) Phylogenetic identification and in situ detection of individual microbial cells without cultivation. Microbiological Reviews 59: 143169

[8] Ravel J, Gajer P, Abdo Z, Schneider GM, Koenig SSK, et al. (2011) Vaginal microbiome of reproductive-age women. Proceedings of the National Academy of Science USA 108: 4680-4687

[9] Srinivasan S, Hoffman NG, Morgan MT, Matsen FA, Fiedler TL, et al. (2012) Bacterial communities in women with bacterial vaginosis: high resolution phylogenetic analysis reveal relationships of microbiota to clinical criteria. PLOS One 7: 6

[10] Zhou X, Bent SJ, Schneider MG, Davis CC, Islam MR, Forney LJ (2004) Characterization of vaginal microbial communities in adult healthy women using cultivationindependent methods. Microbiology, 150: 2565-2573.

[11] Koumans EH, Sternberg M, Carol B, McQuillan G, Kendrick $\mathrm{J}$, et al. (2007) The prevalence of bacterial vaginosis in the United States, 2001-2004; Associations with symptoms, sexual behaviors, and reproductive health. Sexually Transmitted Diseases 34: 864-869.

[12] Amsel R, Totten PA, Spiegel CA, Chen KC, Eschenbach D, Holmes KK (1983) Nonspecific vaginitis. Diagnostic criteria and microbial and epidemiologic associations. American Journal of Medicine 74: 14-22

[13] Nugent RP, Krohn MA, Hillier SL. (1991) Reliability of diagnosing bacterial vaginosis is improved by a standardized method of gram stain interpretation. Journal of Clinical Microbiology 29: 297-301

[14] Beck D and JA Foster (2014, in press) Machine learning techniques accurately classify microbial communities by bacterial vaginosis characteristics. PLoS ONE.

[15] Hickey RJ, Zhou X, Pierson JD, Ravel J, Forney LJ. Understanding vaginal microbiome complexity from an ecological perspective. Transl Res. 2012 Mar 6

[16] Wang O, GM Garrity, JM Tiedje and JR Cole. (2007) Naïve Bayesian Classifier for Rapid Assignment of rRNA Sequences into the New Bacterial Taxonomy. Appl. Environ. Microbiol. 73(16):5261. DOI: 10.1128/AEM.00062-07. 
[17] Madan JC, Salari RC, Saxena D, Davidson L, O'Toole GA, Moore $\mathrm{JH}$, et al. Gut microbial colonisation in premature neonates predicts neonatal sepsis. Arch Dis Child Fetal Neonatal Ed. 2012 May 6.

[18] Madan JC, Koestler DC, Stanton BA, Davidson L, Moulton LA, Housman ML, et al. Serial analysis of the gut and respiratory microbiome in cystic fibrosis in infancy: interaction between intestinal and respiratory tracts and impact of nutritional exposures. mBio. 2012;3(4).

[19] Forney LJ, Foster JA, Ledger W. The vaginal flora of healthy women is not always dominated by Lactobacillus species. J Infect Dis. 2006 Nov 15;194(10):1468-9; authorreply1469-70.

[20] Ravel J, Brotman RM, Gajer P, Ma B, Nandy M, Fadrosh $\mathrm{DW}$, et al. Daily temporal dynamics of vaginal microbiota before, during and after episodes of bacterial vaginosis. studies. 2013;19:20.
[21] Ravel J, Brotman RM, Gajer P, Ma B, Nandy M, Fadrosh $\mathrm{DW}$, et al. Daily temporal dynamics of vaginal microbiota before, during and after episodes of bacterial vaginosis. studies. 2013;19:20.

[22] Ravel J, Personal communications. 2014.

[23] Forney LJ, Gajer P, Williams CJ, et al. (2010) Comparison of self-collected and physician-collected vaginal swabs for microbiome analysis. Journal of Clinical Microbiology 48:1741-1748. doi: 10.1128/JCM.01710-09

[24] Yuan S, Cohen DB, Ravel J, et al. (2012) Evaluation of methods for the extraction and purification of DNA from the human microbiome. PLoS ONE 7:e33865. doi: 10.1371/journal.pone.0033865.t004

[25] Williams, H., Carter, J., W. Campbell, Roy, K., Dozier, G. (to appear). "Genetic \& Evolutionary Feature Selection for Author Identification of HTML Associated with Malware," International Journal of Machine Learning and Computing. 